\title{
Japanese EFL Students' Reading Processes for Academic Papers in English
}

\author{
Yuko Hijikata $^{1}$, Yasuo Nakatani ${ }^{2} \&$ Maki Shimizu ${ }^{3}$ \\ ${ }^{1}$ School of Management, Tokyo University of Science, Saitama, Japan \\ ${ }^{2}$ School of Economics, Hosei University, Tokyo, Japan \\ ${ }^{3}$ Organization for Higher Education and Student Services, Gunma University, Gunma, Japan \\ Correspondence: Yuko Hijikata, School of Management, Tokyo University of Science, Saitama, Japan. Tel: \\ 81-480-21-7647. E-mail: yuko.hijikata@rs.tus.ac.jp
}

Received: September 19, 2012

Accepted: October 24, $2012 \quad$ Online Published: January 16, 2013

doi:10.5539/jel.v2n1p70

URL: http://dx.doi.org/10.5539/jel.v2n1p70

\begin{abstract}
Academic reading has been less emphasized compared with academic writing as a site of research inquiry. Although some studies have examined reading strategy use in academic reading (e.g., Block, 1986; Plakans, 2009), these studies used short passages only, and there have been a few studies that have focused on the mental representation constructed while we read research papers (e.g., Wyatt et al., 1993). Considering that academic discourse has a particular, distinct structure (e.g., Swales, 1990), it is necessary to examine the process of reading academic papers separately from that of reading in general, focusing on the rhetorical particularities of academic discourse. Against this research background, we investigated how Japanese EFL students read English academic papers, focusing on the interactions among L2 proficiency, reading strategies, and the rhetorical features of the papers. We used the following methods: video observation, the "think-aloud protocol," document analysis of notes taken by the participants while reading, and a post-reading interview. The reading strategies identified on the basis of the think-aloud protocol were categorized into local, global, and metacognitive strategies. The main results were as follows. First, the Japanese EFL readers had difficulty recognizing academic discourse. Therefore, they could not read the papers efficiently. Second, the students focused on understanding formulas and figures presented in the documents. Third, although the students used local and global strategies frequently, their purposes in using these strategies varied depending on their L2 competence, their background knowledge about the topic of the paper, and their familiarity with the discipline-specific academic discourse.
\end{abstract}

Keywords: academic reading, English as a foreign language (EFL), reading strategy, think-aloud protocol, genre analysis

\section{Introduction}

\subsection{Second Language Reading and Reading Strategy}

It has been argued that second language (L2) reading is not a single-factor process (e.g., Bernhardt, 2005; Grabe, 2009; Grabe \& Stoller, 2002; Koda, 2005; Nassaji, 2003; Urquhart \& Weir, 1998). Many researchers have distinguished lower-level processing, examples of which include word recognition and syntactic processing, from upper-level or global processing, for instance inference generation or use of background knowledge. One major issue in this area is how extensively these subcomponents are related to each other. Previous studies have examined the relationship between general reading comprehension skill and each subcomponent, finding moderate to high correlations (e.g., Nassaji, 2003; Nassaji \& Geva, 1999; Shiotsu \& Weir, 2007). The other key issue is how to overcome difficulties with each subcomponent. This phase has been examined by means of reading strategy studies. As Table 1 shows, frequently investigated strategies have included translating and/or interpreting the meaning of a word, a clause, or a sentence; paraphrasing or summarizing; rereading; use of background knowledge; recognizing text structure; and self-monitoring of a reader's own comprehension and/or comments on their own reading problems. 
Table 1. Previous studies that have investigated reading strategies using the think-aloud protocol

\begin{tabular}{rllll}
\hline & Study & Participants & Materials \\
\hline 1 & Block & Six English as a second & Two passages, respectively \\
& $(1986)$ & language (ESL) learners (three & composed of 589 words and \\
& & native speakers of Spanish, 843 words taken from an \\
& & three native speakers of & introductory psychology \\
& & Chinese) and three native & textbook \\
& & speakers of English &
\end{tabular}

Types of strategy

[a] General strategies: (1) Anticipate content, (2) Recognize text structure, (3) Integrate information, (4) Question information in the text, (5) Interpret the text, (6) Use general knowledge and associations, (7) Comment on behavior or process, (8) Monitor comprehension, (9) Correct [one's] behavior [Correct one's own incorrect interpretation], (10) React to the text

[b] Local strategies: (11) Paraphrase, (12) Reread, (13) Question the meaning of a clause or sentence, (14) Question the meaning of a word, (15) Solve vocabulary problem

\begin{tabular}{|c|c|c|c|}
\hline 2 & $\begin{array}{l}\text { Block } \\
(1992)\end{array}$ & $\begin{array}{l}25 \text { university students- } \\
16 \text { proficient readers (half L1) } \\
\text { and } 9 \text { less proficient readers ( } 3 \\
\text { L1) }\end{array}$ & $\begin{array}{l}\text { A passage composed of } 589 \\
\text { words taken from an } \\
\text { introductory psychology } \\
\text { textbook }\end{array}$ \\
\hline 3 & ( & $\begin{array}{l}\text { (1) L2-Intermediate }(n=20), \\
\text { (2) L2-Advanced }(n=20),(3) \\
\text { L1-Japanese }(n=20), \\
\text { L1-English }(n=16)\end{array}$ & $\begin{array}{l}\text { Two short stories from } \\
\text { Japanese storybook }\end{array}$ \\
\hline 4 & $\begin{array}{l}\text { Kamhi- } \\
\text { Stein } \\
(2003)\end{array}$ & $\begin{array}{l}\text { Four bilingual, } \\
\text { native-Spanish-speaking college } \\
\text { students }\end{array}$ & $\begin{array}{l}\text { An English text (850 words) } \\
\text { and a Spanish text }(655 \\
\text { words })\end{array}$ \\
\hline
\end{tabular}

[a] Evaluation: (1) Recognize problem, (2) Identify source

[b] Action: (1) Plan, (2) Act

[c] Check: (1) Check solution, (2) Revise solution

(1) Graphomorphemic/ Graphophonemic analysis, (2) Word recognition, (3) Syntactic/Semantic analysis of sentence, (4) Backward inference, (5) Elaborative inference, (6) Predictive inference, (7) General knowledge and association, (8) Comments on text structure, (9) Comments on own behavior, (10) Other comments

(1) Mentally translating, (2) Detecting comprehension problems, (3) Attempting to solve comprehension problems, (4) Paraphrasing, (5) Using context, (6) Recognizing text structure, (7) Rereading, (8) Recognizing important information, (9) Summarizing important information, (10) Integrating information, (11) Making inferences about the text, (12) Questioning the text, (13) Making predictions, (14) Reacting affectively

5 Dewey (1) 15 learners of Japanese as a Three Japanese reading

(2004) second language studying passages of approximately (1) Analyze to identify meaning, (2) Comment on text structure, (3) Translate, (4) abroad, (2) 15 learners of Japanese in intensive domestic immersion

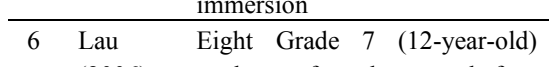

(2006) students-four boys and four girls-from two secondary schools in Hong Kong
350-500 characters

(1) Narrative text and (2) Expository text. Each text is followed by four open-ended questions. (1) is a 550-word fable chosen from a Chinese storybook written for Hong Kong students, while (2) is a 500-word informational article about two different Hong Kong people's ancestors, chosen from a similar-level history book for Hong Kong students. Paraphrase or summarize, (5) Read text aloud, (6) Comment on task, (7) Use or reference prior knowledge of experience, (8) Monitor understanding (or comprehension monitoring), (9) Make inferences, (10) Show affective reaction (text)

[a] Pre-reading strategy: (1) Set up reading goal; (2) Make an overview of the text to activate prior knowledge or generate an initial hypothesis about the text; (3) Make use of the relevance signals of the text, such as title, paragraphs, graphic signals etc., to form an initial understanding of the text

[b] Strategy for identifying and interpreting the main information: (4) Paraphrase parts of text into oral language or more familiar terms; (5) Look for key words, topic sentences and topic paragraphs; (6) Summarize main ideas after reading each part of the text; (7) Skip irrelevant or unimportant words or sentences; (8) Construct a macrostructure of the text based on prior knowledge; (9) Flexibly adjust the hypothesized macrostructure based on newly encountered information in the text; (10) Predict content/structure of the text; (11) Generate questions about the main ideas and try to find out the answers; (12) Make use of text structure to understand or organize the main ideas; (13) Compare different main ideas, (14) Make visual images based on the description of the text; (15) Make elaborative inferences to interpret the content of the text, such as relationships among the characters, characteristics of the characters, cause and effects of the incidents, etc.; (16) Make elaborative inference to achieve in-depth understanding of the theme; (17) Generate elaborations of the main ideas; (18) Make affective reactions to the text

[c] Reading monitoring strategy: (19) Adjust the reading speed based on the level of difficulty; (20) Read selectively and flexibly in accordance with the reading goal; (21) $\mathrm{Be}$ aware of the problems encountered in the text; (22) Be aware of personal difficulties in comprehending the text; (23) Be aware of and revise the mistakes arising during the reading process or when answering the questions; (24) Reread the difficult parts to resolve comprehension difficulties; (25) Look backward or ahead to see if there is information in the text that can resolve the comprehension difficulties; (26) Make use of contextual cues to interpret difficult words; (27) Infer the meaning of difficult words based on their structural features; (28) Infer the meaning of difficult words based on their pronunciation

[d] Post-reading strategy: (29) Construct cohesive summary of the text; (30) Evaluate and reconstruct the hypothesized macrostructure of the text; (31) Reread parts of the text to increase memory of it, facilitate question answering, or clarify unclear parts; (32) Evaluate the content of the text or the quality of writing; (33) Make affective reactions to the overall content of the text

Several reading strategy studies have focused on the differences between L1 and L2 reading strategies (Block, 1986, 1992; Carrell, 1989; Horiba, 1996; Kamhi-Stein, 2003) and the relationship between L2 competency and reading strategy use (e.g., Dewey, 2004; Horiba, 1996; Lau, 2006). It has been shown that proficient readers tend 
to use Global Strategies while less proficient readers tend to use Local Strategies, especially at the word level (Horiba, 1996; Lau, 2006; Plakans, 2009). Reading strategies in this context have been assessed mainly by questionnaire (e.g., Carrell, 1989; Cogmen \& Saracaloglu, 2009; Phakiti, 2003) and the think-aloud protocol. Especially since the 1990s, more studies have used the think-aloud protocol to examine reading strategy (see Table 1).

Although we can induce much information through the think-aloud protocol (as well as other measures), this protocol also has limitations, including the following (see Ericsson \& Simon, 1984, for the details). First, it can measure only strategies that can be verbalized, whereas eye tracking and reaction time measurement, for instance, are likely to reflect automatic, unintentional processes. Second, we have to admit that the think-aloud protocol leads to large individual differences. Some readers may not find any difficulty in verbalizing what they are thinking, while others may have no idea how to verbalize what they have in their mind, or just forget to do so if they concentrate on the primary task. We need to consider these weaknesses of the think-aloud protocol, choose appropriate tasks for verbalization, and compensate for the variation in the protocols used by participants with a post-interview.

\subsection{Strategy in Academic Reading}

Compared with reading in English for general purposes, reading in English for academic purposes (EAP) has the following features. First, the assumption is generally made that readers have some background knowledge of the topic. Of course, writers may tailor their writing to their audience differently depending on whether readers are likely to be, for example, freshmen, graduate students, or established researchers. However, readers are still supposed to interpret the messages in the text by activating their background knowledge related to the topic (to a greater or lesser degree). Second, academic reading, especially reading academic papers, requires understanding of specific features of rhetoric. A particularly useful concept influenced by rhetorical theory for students learning about academic reading and writing is genre analysis (e.g., Swales, 1990), which analyzes passages from the perspective of genre as identified by discourse features.

Swales (1990) focused on the effects and usages of rhetorical features in EAP discourse and developed genre analysis, which is a rhetoric strategy for the communication of a specific message in a way that makes the passage easier to read. A "move" in the genre analysis is defined as "a discoursal or rhetorical unit that performs a coherent communicative function in a written or spolen discourse" (Swales, 2004, p. 228). Move 1 identifies or reviews (reminds the reader of) the topic, Move 2 identifies gaps in the state of present research ("establishes a niche" for the work being presented), and Move 3 states the outline or major findings of a study. Each move is further divided into subcategories: Move 1A (Claiming centrality), Move 1B (Making topic generalization[s]), Move 1C (Reviewing items of previous research), Move 2A (Counter-claiming), Move 2B (Indicating a gap), Move 2C (Question-raising), Move 2D (Continuing a tradition), Move 3A (Outlining purposes), Move 3B (Announcing present research), Move 3C (Announcing principal findings), and Move 3D (Indicating RA [research article] structure). As Swales (2004) claimed, ESL and EFL learners trying to work with EAP need to learn such moves or discourse strategies.

Although researchers have recognized the importance of reading strategy in EAP, the number of studies that have focused on this area is limited. Reading academic papers or textbooks is an essential skill for researchers, regardless of whether English is their L1 or L2. Several types of reading skills are required for EAP, including reading for the gist, reading to include the paper in a literature review, and reading to evaluate the research.

The reading process engaged in when dealing with academic papers by professional scholars working in their L1 has been investigated by Wyatt et al. (1993) and Paul and Charney (1995). Both studies employed think-aloud protocols; the main difference between them was the materials used. Wyatt et al. asked 15 active social scientists to choose papers with reference to their research interests, while in Paul and Charney's study, 12 scholars were provided with the introduction section of papers on chaos theory, a major theory of physical systems developed in the 1960s.

Wyatt et al. (1993) required the participants to read as they always did, and classified their reading processes into the following categories: (a) strategy use (predict, look, jump forward, jump backward, jump back and forth, backtrack, vary reading style, attend to tables/figures, construct paraphrases, construct conclusions); (b) monitoring (note difficulty, note what is known, evaluate relevance); and (c) evaluation (react evaluatively based on one's own knowledge, react based on one's personal prior knowledge, evaluate the text, [show] negative affective reaction, express interest). They showed that the comprehension strategies used by the participants were related to the self-regulated nature of the reading. In particular, the participants read to determine whether the texts were worth reading and whether the descriptions were reliable-in fact, their monitoring and salient 
evaluative reactions were closely related. Therefore, Wyatt et al. claimed that experts' EAP reading processes involve both "cold" literal and "hot" interpretive-evaluative processing. In other words, processing in which readers actively interact with text is called "hot processing" and processing in which readers understand the text literally is "cold processing."

Paul and Charney (1995) explored whether there was any difference in introductory moves used in the chaos theory papers between the period when chaos theory was at an early stage of development (papers published in 1975 and 1978) and when it had been widely acknowledged (papers published in 1989 and 1990). Their study focused on the introduction section of the papers in order to examine the awareness of writers' use of the rhetoric features by readers who were established researchers in a field. They found that regardless of the stage of development of chaos theory, the readers' level of interest was influenced by how much they felt the theory could be related to their own research. Moreover, the readers paid most attention to how the new theory was presented when they were reading parts of the latest papers exhibiting features of Moves 1 and 2. As well, even if readers were negative toward chaos theory, they tended to leave comments on Move 3, the part that describes areas unexplored by previous studies.

L2 readers' strategies for reading academic materials were examined by Li and Munby (1996), who looked at the reading processes of two ESL graduate students, using a think-aloud protocol. The materials employed were textbooks and papers, selected by the participants. Their results showed that the participants frequently used strategies such as translation, background knowledge use, self-questioning, and finding topic sentences. Li and Munby's study has provided us with many insights into academic reading processes; however, there are two caveats which we have to consider. First, the automatization of processing is different between ESL and EFL learners. Li and Munby's participants were advanced learners whose TOEFL scores were above 610, meaning that they were highly proficient. However, most L2 readers, especially in the EFL environment, have much lower English proficiency. In Japan, where English is studied as a foreign language, many researchers have difficulties reading and writing papers in English but nevertheless have to use English in order to keep up with current research or publish their findings. Therefore, we need to confirm whether results similar to $\mathrm{Li}$ and Munby's are obtained for less proficient ESL and EFL readers before we attempt to apply these research outcomes to teaching. In short, it is important to examine the behavior of EFL participants. Second, Li and Munby's coding framework did not include attention to the formal features of academic discourse, which we believe play an essential role in academic reading. Even if academic authors attempt to write in an objective way, they resort to certain conventional rhetorical and stylistic features to convey their meaning. The next section explains how these features are incorporated into academic papers.

\subsection{Rhetoric in Academic Papers}

A reader-centered approach is considered effective in English writing. Writers need to convey information in a way such that readers can clearly follow and understand their intention. It is also important to consider how best to attract readers' attention and guide them appropriately. To achieve these goals, it is claimed that coherence and cohesion should be developed to organize the flow of arguments and combine ideas in a paragraph in English texts (Halliday \& Hasan, 1976). Coherence here is defined as a certain kind of semantically informed relationship among the elements of a text-specifically, relationships based on logical or natural thematic development, organization of information, or the communicative purpose of the particular discourse (Halliday, 2004). Cohesion, in contrast, refers to the grammatical or lexical relationships between the different elements of a text; it helps readers to read smoothly by strengthening the connections between sentences (Johnson, 1992). It is generally agreed that enhancing coherence and cohesion contributes to readability by allowing faster processing of text and easier access to the main purposes of text (Halliday, 1994; Moore, 2006)

\subsection{Genre-Based Instruction and Rhetorical Awareness}

There have been studies that have examined the effects of students' genre analyses in EAP writing classes (e.g., Cheng, 2008; Negretti \& Kuteeva, 2011). Cheng (2008) chose a native-Chinese-speaking graduate student as a focal subject, analyzing the student's discipline-specific genre exemplars and finding two prominent types of features: (a) rhetorical, as evidenced in the student's consistent attention not only to the generic features of the text, but also to the underlying rhetorical parameters, such as reader, writer, and purpose and (b) evaluative, as shown in the student's increasingly sophisticated evaluation of the discourse-level generic features of the genre exemplars. Cheng emphasized that genre-based instruction was effective in promoting academic literacy among L2 students.

Negretti and Kuteeva (2011) conducted a case study with eight participants in an EAP class who were native speakers of Spanish. They used the following data sources: observation, students' reflections on seminar 
activities, a textual analysis of online tasks and final assignments. They concluded that fostering metacognitive skills helped the participants recognize and use genre features in an appropriate way.

However, it has not been clarified whether genre analyses will affect the process of reading academic papers in the same way as they do for writing. In order to observe L2 readers' natural processes, it is important to examine how these readers identify genre in a reading task.

\subsection{Summary}

As reviewed above, the quantity of academic reading research is small compared with that on academic writing, and we need more research on L2 academic reading. In particular, psycholinguistic research, such as think-aloud protocol research, in this area is very limited. Although a few studies have examined the L2 EAL reading process, using a think-aloud protocol method (e.g., Block, 1986), the materials used in these studies were short passages, not full research papers. Considering the features of academic discourse and the centrality of the research paper as a way of presenting findings, we need to investigate the process of reading academic papers and the strategies used in this process by L2 readers. In particular, novice researchers such as master's-level students may have difficulties in reading academic papers, since in addition to having less familiarity with the field they are likely to have less familiarity with the features of academic discourse than older researchers. Accordingly, we examine the academic reading process used by Japanese master's students in an EFL context.

\subsection{The Present Study}

One purpose of this study is to examine whether EFL learners' L2 competence affects their discipline-specific reading processes. The other is to clarify the relationship between the common rhetorical features of academic discourse and EFL learners' reading processes. Based on the literature review above, we propose two hypotheses. Hypothesis 1 is developed based on previous studies of L2 reading strategy that have shown differences in strategy use between proficient and less proficient readers (e.g., Horiba, 1996; Lau, 2006). Hypothesis 2 is based on Paul and Charney (1995), who claimed that their participants tended to leave comments on sections of the papers they read that described areas unexplored by previous studies or outlined the purposes of the study. Although Paul and Charney targeted professional researchers whose L1 was English, their study is the greatest influence on ours methodologically, in that they used the think-aloud protocol and examined the relationship between readers' reactions to the paper and the paper's rhetorical features.

Hypothesis 1: When reading an academic paper, a proficient reader uses Global Strategies rather than Local Strategies, while a less proficient reader uses Local Strategies rather than Global Strategies.

Hypothesis 2: EFL learners frequently use Global Strategies for Move 3 (outline/major findings) but not in Moves 1 (topic/review) or 2 (gap).

\section{Method}

Two undergraduate students at the School of Management at a private university in Japan participated in this study. Both of them had completed their graduation theses and intended to start their master's programs. The TOEIC score of Participant 1 was 300, while the TOEFL-PBT score of Participant 2 was 580. Although it is difficult to accurately convert TOEIC and TOEFL scores, the 300 level in TOEIC is considered to represent a beginning to the intermediate level of English proficiency, while TOEFL-PBT 580 is a score which satisfies the entry requirements of many undergraduate programs in English-speaking countries. Thus, it is clear that Participant 2 was much more proficient in English. Participant 2 had studied in the U.S. for a year, and had had extensive English instruction there, including courses in academic reading and writing. Participant 1 was a more typical student in a Japanese EFL environment. He had never studied in an English-speaking country and had difficulty reading English papers, although it was a necessary task for his studies. Both students were in their early 20 s.

For the current study, the participants selected papers based on their graduation topics. Participant 1 read an article on statistics (Prentice \& Pyke, 1979), while Participant 2 chose an article on economics (Gilbore, 2010). Neither of the papers included experimental research; the statistics paper included several formulas, while the economics paper was quite descriptive. Neither of the participants had read either paper previously. To control for text-related variables, it would seem preferable to have the participants read the same paper, and indeed, this research design might improve the reliability of the methods used. However, the focus of this study is to explore the qualitative implications of the cognitive behaviors of EFL learners who do not have specific training. Rather than providing the materials ourselves, which could potentially have caused problems stemming from the participants' lack of background knowledge or from anxiety, we accepted any paper the participants brought. Indeed, using different reading materials per participant in one study to obtain qualitative data is not unusual in the 
area of English for academic purposes (e.g., Cheng, 2008; Wyatt et al., 1993).

We collected data using the following methods: think-aloud protocols, videorecordings, pre- and post-interviews, and notes taken by the students while they read the papers. The think-aloud protocols were recorded using an IC recorder and transcribed. During the reading sessions, the students were able to look up unfamiliar words in a dictionary, underline or highlight significant statements in the paper, and take notes as they usually did. The verbal protocol was recorded while the participants were reading through the paper. The reading time for the protocol was 5.5 hours for Participant 1 and 2.5 hours for Participant 2. The transcriptions of the sections of the protocols dealing with the papers' abstract, keyword, and introduction sections were segmented by the two authors of this paper, following Green's (1998) examples. Disagreements about the segmentation units were resolved through discussion.

Reading strategies were categorized with reference to the frameworks used by Plakans (2009) and in our pilot study (Hijikata, Nakatani, \& Shimizu, 2011). The first category was Local Strategies, composed of Rereading, Breaking lexical items into parts/using phonological cues, and Guessing and interpreting the meaning. The latter two strategies were divided into word, phrase, sentence, formula, and notion levels. The second category was Global Strategies, including Skimming for gist, Asking questions, Recognizing text structure/rhetorical cues, Identifying/summarizing main ideas, and Responding to text with personal experience/opinions. The third category was Metacognitive Strategies, which included Recognizing lack of comprehension and Confirming understanding (See Table 2).

Table 2. Framework of this study

\begin{tabular}{|c|c|c|c|c|c|c|c|}
\hline & Strategy Type & Subtypes & $\mathrm{W}$ & $\mathrm{Ph}$ & $\mathrm{S}$ & $\mathrm{F}$ & $\mathrm{N}$ \\
\hline \multirow[t]{2}{*}{ A } & Local Strategies & Rereading & & & 1 & & \\
\hline & & $\begin{array}{l}\text { Breaking lexical items into parts/using phonological cues } \\
\text { Guessing the meaning, Interpreting the meaning }\end{array}$ & 2 & 3 & 4 & 5 & 6 \\
\hline \multirow[t]{4}{*}{ B } & Global Strategies & $\begin{array}{l}\text { Skimming for gist } \\
\text { Asking questions }\end{array}$ & & & $\begin{array}{l}7 \\
8\end{array}$ & & \\
\hline & & Recognizing text structure/rhetorical cues & & & 9 & & \\
\hline & & Identifying/summarizing main ideas & & & 10 & & \\
\hline & & Responding to text with personal experience/opinions & & & 11 & & \\
\hline \multirow[t]{2}{*}{$\mathrm{C}$} & Metacognitive & Recognizing lack of comprehension & & & 12 & & \\
\hline & Strategies & Confirming understanding & & & 13 & & \\
\hline
\end{tabular}

Note. $\mathrm{W}=$ word(-level processing), $\mathrm{Ph}=$ phrase, $\mathrm{S}=$ sentence, $\mathrm{F}=$ formula, $\mathrm{N}=$ notion.

One of the aims of this study was to examine the relationship between the reading strategies adopted and the content of the academic discourse. Therefore, the reading strategy analysis was combined with move analysis. The introduction section of each paper was segmented from the (move-based) genre perspective (Swales, 1990, 2004), and the participants' reading strategies were compared by move. Additionally, in order to clarify the nature of the readers' explicit knowledge of the structural, rhetorical, and genre features of the texts and their own use of strategies, this paper gathered and presents interview results.

\section{Results and Discussion}

\subsection{The Relationship between L2 Proficiency and Reading Strategies}

One of the focuses of this study was to see whether the two participants used similar reading strategies. The instances of reading strategies (as distinct from separate strategies) for the introduction sections were 302 for Participant 1 and 24 for Participant 2. We compared their strategies by estimating the percentage of total strategy use of each strategy. The details are shown in Table 3, and integrated results are shown in Figure 1. 
Table 3. Reading strategies used by each participant

\begin{tabular}{|c|c|c|c|c|c|c|c|}
\hline & \multirow[b]{2}{*}{ Strategy } & \multicolumn{3}{|c|}{ Participant 1} & \multicolumn{3}{|c|}{ Participant 2} \\
\hline & & $\mathrm{N}$ & $\%$ & & $\mathrm{~N}$ & $\%$ & \\
\hline \multirow{5}{*}{ A } & Rereading & 19 & 6.29 & \multirow{6}{*}{75.17} & 1 & 4.17 & \multirow{6}{*}{20.84} \\
\hline & Word & 59 & 19.54 & & 1 & 4.17 & \\
\hline & Phrase & 103 & 34.11 & & 0 & 0.00 & \\
\hline & Sentence & 20 & 6.62 & & 3 & 12.50 & \\
\hline & Formula & 23 & 7.62 & & 0 & 0.00 & \\
\hline & Notion & 3 & 0.99 & & 0 & 0.00 & \\
\hline \multirow{5}{*}{ B } & Skimming & 0 & 0.00 & \multirow{5}{*}{17.21} & 1 & 4.17 & \multirow{5}{*}{79.17} \\
\hline & Asking questions & 0 & 0.00 & & 0 & 0.00 & \\
\hline & Recognizing text structure & 3 & 0.99 & & 6 & 25.00 & \\
\hline & Summarizing main ideas & 27 & 8.94 & & 5 & 20.83 & \\
\hline & Responding & 22 & 7.28 & & 7 & 29.17 & \\
\hline \multirow{3}{*}{$\mathrm{C}$} & Lack of comprehension & 15 & 4.97 & \multirow{2}{*}{7.62} & 0 & 0.00 & \multirow{2}{*}{0.00} \\
\hline & Confirming understanding & 8 & 2.65 & & 0 & 0.00 & \\
\hline & Total & 302 & & 100 & 24 & & 100 \\
\hline
\end{tabular}

Note. A = Local Strategies, $\mathrm{B}=$ Global Strategies, $\mathrm{C}=$ Metacognitive Strategies. $\%=$ the percentage of each participant's total number of uses of all strategies accounted for by this strategy.

Table 3 shows that the Participant 1 was observed using 227 Local Strategies, accounting for $75.17 \%$ of his strategy use, and used Global Strategies 52 times, accounting for $17.21 \%$ of his total. Metacognitive Strategies were found in only 23 cases, covering $7.62 \%$ of his strategy use. Participant 2, on the other hand, used Global Strategies the most-19 times out of 24 , accounting for $79.17 \%$ of his strategy use-followed by Local Strategies, accounting for five cases and $20.83 \%$.

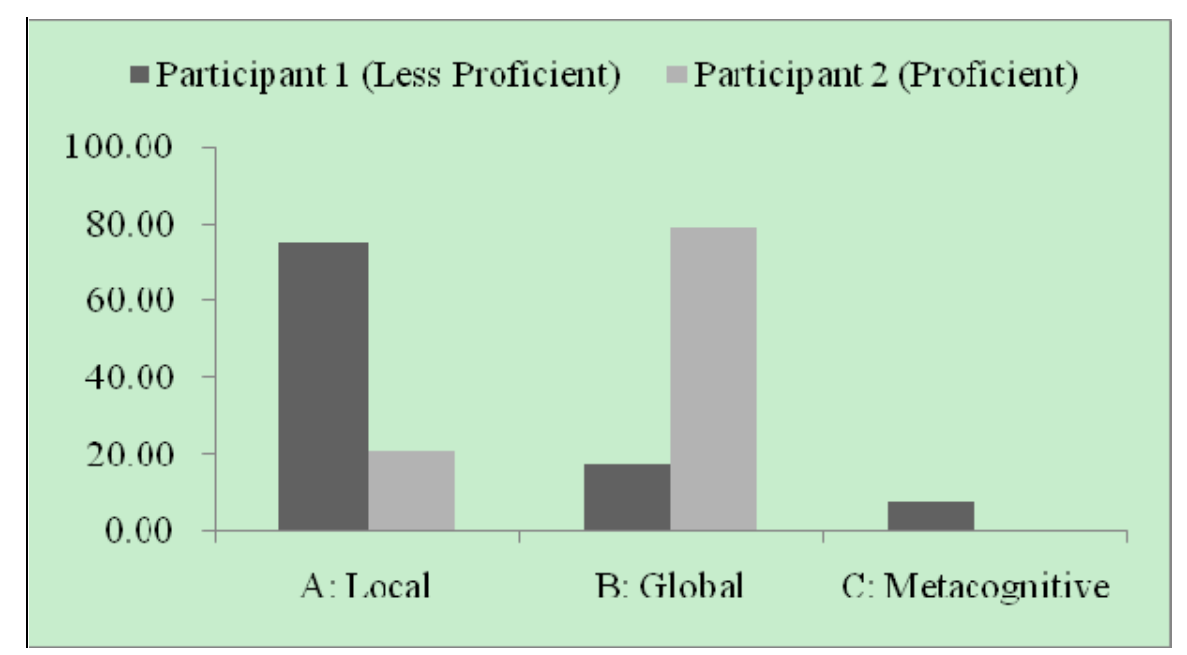

Figure 1. Overall breakdown of strategies used by the two participants

As Figure 1 illustrates, Participant 2 used Global Strategies (79.17\%) more than Local Strategies (20.84\%), while Participant 1 used Local Strategies the most (75.17\%). Metacognitive Strategies were used only by Participant 1 (7.62\%). The components of the Local Strategies, as given above, are illustrated in Figure 2. 


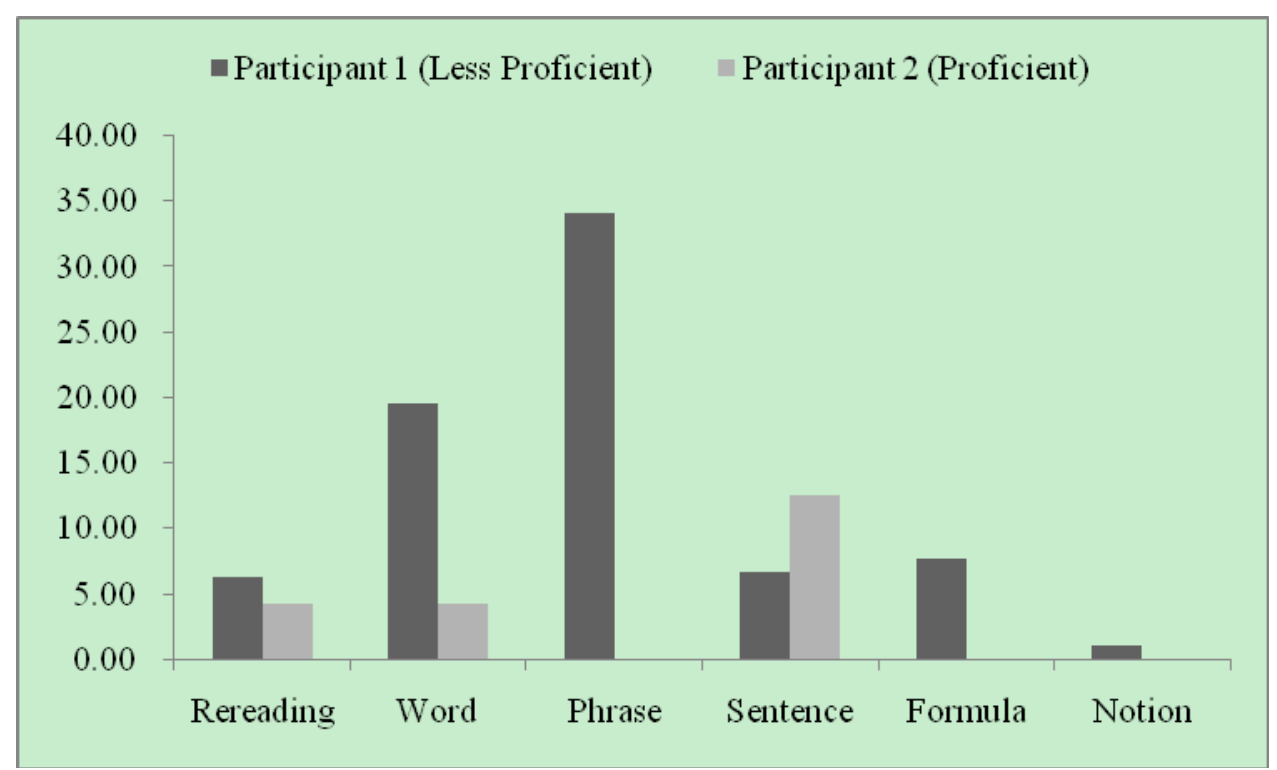

Figure 2. Subcategories in local processing

When we consider the subcategories of the Local Strategies used, we see that the strategy most frequently used by Participant 1 was Phrase-level processing (34.11\%), followed by Word-level processing (19.54\%). Therefore, it is clear that Participant 1 interpreted each sentence as a small unit. Another important feature of this participant's reading strategy was that Formula-level processing accounted for $7.62 \%$ of all strategy uses, making it more frequent than Sentence-level processing (6.62\%). This participant said in the post-interview that he had problems reading and thus that he tended to use formulas in order to understand the author's intention clearly. In contrast, P-*articipant 2 used Sentence-level processing the most $(12.50 \%)$.

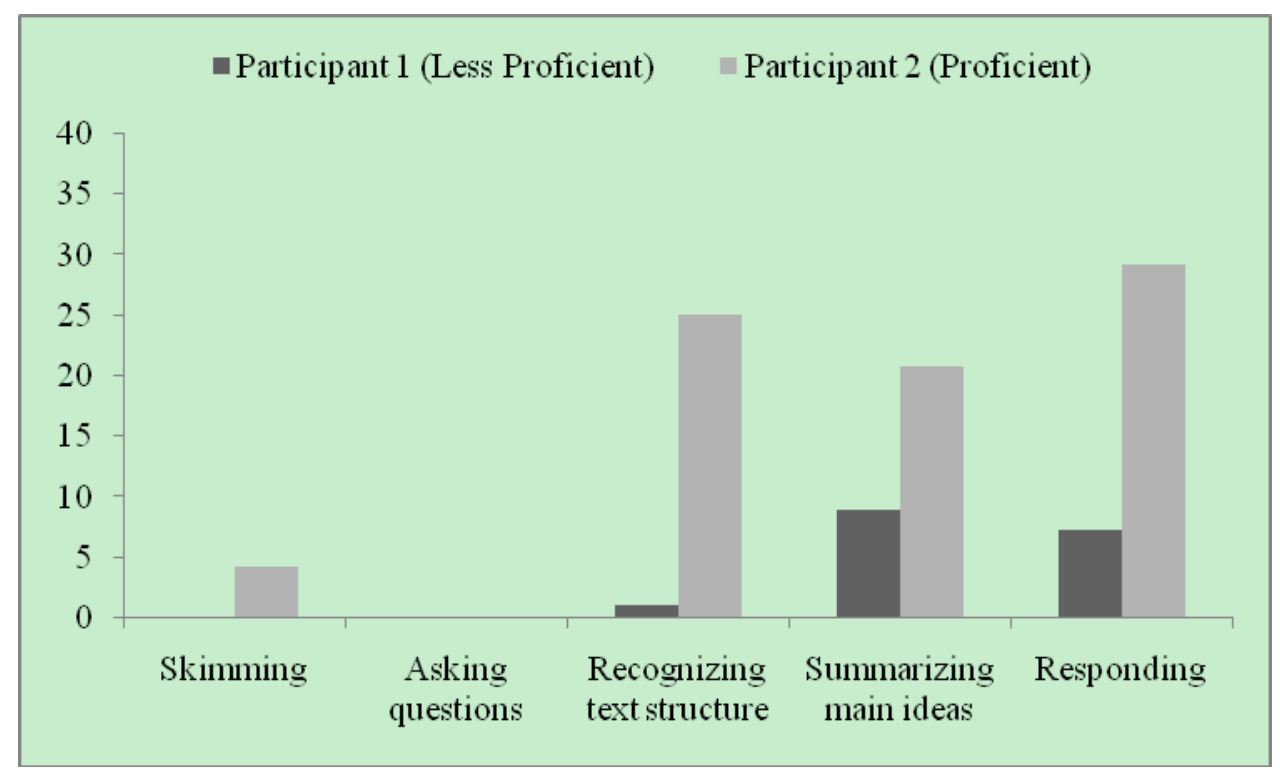

Figure 3. Global processing

In the Global Strategy framework, the strategy most frequently used by Participant 1 was Summarizing main ideas $(8.94 \%)$. It is worth noting here that this participant tended to interpret at the word level first, followed by the phrase level and then the sentence level; finally, he summarized the main ideas. In other words, Participant 1 processed most parts of the paper four times. However, he did not pay attention to the structure of the text. 
Although he made a few comments about sectioning, he kept reading in the same order and seldom went back to previous sections.

In contrast, Participant 2 was very sensitive to the text structures. Before he started reading the article, he checked the section titles and reviewed the structure of the paper. In addition, he read through the paper by skimming the content of one or two paragraphs.

Responding was a strategy utilized by both readers. However, their purposes in using this strategy differed. Participant 1 tried to understand specific statistical formulas in his paper by applying case examples, such as the rate of becoming ill from smoking. This was clear from the notes he made during his reading. On the other hand, Participant 2 made inferences from the content by activating his background knowledge about the author's ideas.

\subsection{The Relationship between Moves and Reading Strategies}

The second focus of this paper is the relationship between moves and reading strategy, which has rarely been examined in previous studies. Among moves, the proportion related to Move 3 was the highest (.575 for Participant 1, .530 for Participant 2), followed by Move 1 (.425 for Participant 1, .294 for Participant 2). Neither of the participants used many strategies for Move 2; Participant 1 did not use any strategy at all for Move 2 and Participant 2 did only at the proportion of .176. This breakdown is also affected by the structure of the papers the participants read, since different papers can use each move with different degrees of prominence. The papers that the participants in this study read did not include many features of Move 2, and unsurprisingly, the total number of strategies used for Move 2 was very small. Therefore, the following analyses focus on Moves 1 and 3. Table 4 and Figure 4 show a comparison between Move 1 and Move 3.

Table 4. Relationship between move and strategy $($ total $=1.00)$

\begin{tabular}{lcccccc}
\hline & \multicolumn{3}{c}{ Move 1 } & \multicolumn{3}{c}{ Move 3 } \\
\hline & A: Local & B: Global & C: Meta & A: Local & B: Global & C: Meta \\
\hline Participant 1 & .303 & .095 & .027 & .466 & .077 & .032 \\
Participant 2 & .059 & .235 & .000 & .059 & .471 & .000 \\
\hline
\end{tabular}

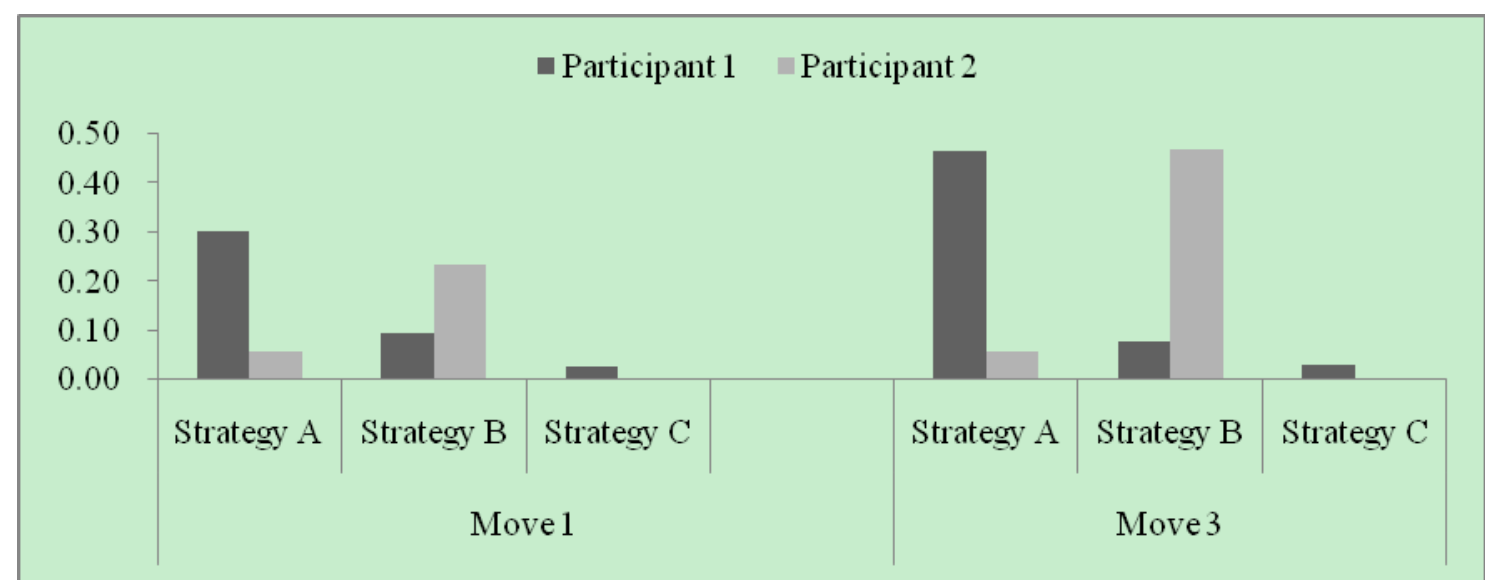

Figure 4. Relationship between moves and strategy

Note. Strategy A = Local Strategies, Strategy B = Global Strategies, Strategy C = Metacognitive Strategies. Move 1 = Topic/review, Move 3 = Outline/major findings.

As Table 4 and Figure 4 show, both participants frequently used the same strategies across different moves; Participant 1 used Local Strategies more, while Participant 2 used Global Strategies more. In order to see the details, we more closely examined the data to see which strategy was used in which subcategory of move. Tables 5 and 6 show the number of strategies that the readers used for each move of the paper. Strategy use for each move by Participant 1 is shown in Table 5 and that by Participant 2 in Table 6 . 
Table 5. Strategy use for each move by Participant 1

\begin{tabular}{lrrrrrrrrrrrrrr}
\hline & \multicolumn{1}{c}{ A: Local Strategies } & \multicolumn{4}{c}{ B: Global Strategies } & \multicolumn{4}{c}{ C: Metacognitive Strategies } \\
\hline & S1 & S2 & S3 & S4 & S5 & S6 & S7 & S8 & S9 & S10 & S11 & S12 & S13 & Total \\
\hline 1A & -- & .02 & .04 & .01 & -- & -- & -- & -- & -- & .01 & -- & .01 & -- & .09 \\
1B & .02 & .07 & .05 & .01 & -- & -- & -- & -- & -- & .01 & .03 & .01 & -- & .20 \\
$1 \mathrm{C}$ & .01 & .03 & .04 & -- & -- & -- & -- & -- & -- & .01 & .02 & -- & -- & .13 \\
\hline 3A & -- & -- & -- & -- & -- & -- & -- & -- & -- & -- & -- & -- & -- & -- \\
3B & .02 & .05 & .15 & .03 & .10 & .01 & -- & -- & -- & .03 & .04 & .02 & .01 & .45 \\
3C & -- & .02 & .06 & .02 & -- & -- & -- & -- & -- & .01 & -- & -- & -- & .13 \\
3D & -- & -- & -- & -- & -- & -- & -- & -- & -- & -- & -- & -- & -- & -- \\
\hline Total & .05 & .19 & .34 & .07 & .10 & .01 & -- & -- & -- & .08 & .09 & .05 & .01 & 1.00 \\
\hline
\end{tabular}

Note. Total $=1.00 . \mathrm{S} 1=$ Rereading, S2 $=$ Word, S3 $=$ Phrase, S4 $=$ Sentence, S5 = Formula, S6 = Notion, S7 $=$ Skimming, S8 = Asking questions, S9 = Recognizing text structure, S10 = Summarizing main ideas, S11 = Responding, S12 = Lack of comprehension, S13 = Confirming understanding.

As can be seen in Table 5, Participant 1 used Local Strategies (S1-S6) more often for both Move 1 and Move 3. In particular, he used Phrase-level processing (S3), in the (sub-)move of "Announcing the present research" (Move 3B). This may be because he was aware that this part was very important and thus made more effort than usual to understand the meaning deeply.

Table 6. Strategy use in each move by Participant 2

\begin{tabular}{rrrrrrrrrrrrrrr}
\hline \multicolumn{1}{c}{ A: Local Strategies } & \multicolumn{4}{c}{ B: Global Strategies } & \multicolumn{3}{c}{ C: Metacognitive Strategies } \\
\hline & S1 & S2 & S3 & S4 & S5 & S6 & S7 & S8 & S9 & S10 & S11 & S12 & S13 & Total \\
\hline 1A & -- & -- & -- & .06 & -- & -- & -- & -- & - & -- & .06 & -- & - & .12 \\
1B & -- & -- & -- & -- & -- & -- & -- & -- & -- & -- & .06 & -- & -- & .06 \\
1C & -- & -- & -- & -- & -- & -- & -- & -- & -- & -- & .12 & -- & -- & .12 \\
\hline 3A & -- & -- & -- & -- & -- & -- & -- & -- & .06 & -- & .18 & -- & -- & .24 \\
3B & -- & -- & -- & -- & -- & -- & -- & -- & -- & -- & .06 & -- & -- & .06 \\
3C & .06 & -- & -- & -- & -- & -- & -- & -- & .12 & -- & .06 & -- & -- & .24 \\
3D & -- & -- & -- & -- & -- & -- & -- & -- & -- & -- & -- & -- & -- &.- \\
\hline Total & .06 & -- & -- & .06 & -- & -- & -- & -- & .18 & -- & .71 & -- & -- & 1.00 \\
\hline
\end{tabular}

Note. Total $=1.00 . \mathrm{S} 1=$ Rereading, S2 $=$ Word, S3 = Phrase, S4 = Sentence, S5 = Formula, S6 = Notion, S7 $=$ Skimming, S8 = Asking questions, S9 = Recognizing text structure, S10 = Summarizing main ideas, S11 = Responding, $\mathrm{S} 12$ = Lack of comprehension, $\mathrm{S} 13=$ Confirming understanding.

As Table 6 indicates, Participant 2 also used the largest proportion of strategies for Move 3 (.54). In particular, he frequently used strategies for $3 \mathrm{~A}$ (Outlining purposes) and $3 \mathrm{C}$ (Announcing principal findings). Another thing we should emphasize is that he used Strategy 11, Responding, more for Moves 1C (Reviewing items of previous research) and 3A (Outlining purposes) than for the other moves. In other words, he read these parts of the article critically, using his prior knowledge.

\subsection{Readers' Awareness of the Special Requirements of Reading Academic Papers}

In this section, the excerpts from the post-interview are introduced to complement the results of the think-aloud protocols. Participant 1 commented on the issue of understanding the formulas as follows (for all excerpts, the authors have translated them from Japanese into English):

I cannot understand English well. Thus, I pay close attention to the formulas. The point is to infer the content of the paper from the formulas.

Since he was not confident that he would understand what the paper reported by reading the text, he was trying to understand the content of the paper through the formulas instead, since these are consistent across languages. 
This corresponds to what Hijikata and Nakatani (2010) have indicated, namely that science students paid more attention to graphs and notes on the graphs than they did to the text. The following is what Participant 2 said after he had finished reading.

I became aware that every paper has similar organization or development. Thus, I have now changed how much I read intensively; I read some parts intensively and other parts roughly. Now, I am aware which part of a paper I am reading and whether the part I am reading now should be understood fully. I took reading and writing courses in the U.S. Although I still follow the models I learned when writing, reading should be taught through practice.

As Participant 2 indicates, he paid attention to the organization of the paper he was reading. Furthermore, he consciously applied what he had learned in his academic writing courses when reading. Although some studies (e.g., Chen, 2008) have examined the effects of genre instruction on the development of rhetorical awareness, most studies in this area have collected their data from students' analyses of the discourse-level generic features in discipline-specific genre exemplars. It is unclear whether readers really became aware of rhetorical structures when a genre analysis task is not required. The results of the present study indicate that the genre-based instruction could affect differences in the reading processes used for academic papers.

\section{Conclusion}

This study examined what types of reading strategies Japanese EFL students at the university level use when they read academic papers in English. In particular, we focused on the effects of learners' L2 proficiency and their understanding of the presence and function of "moves" in academic papers on their use of reading strategies. Data was collected using the think-aloud protocol, video observation, notes that the participants took while reading the paper, and post-interviews. The results can be summarized as follows.

First, the two participants generally used different strategies. The less proficient reader mainly employed Local Strategies, while the more proficient reader frequently used Global Strategies. Among the Local Strategies, the less proficient reader engaged most frequently in word- or phrase-level processing. In contrast, the proficient reader did more sentence-level processing. The less proficient reader used Global Strategies in order to infer what the author had wanted to convey, by using his background knowledge, while the proficient reader used Global Strategies more often to summarize the main ideas in the papers. These results are consistent with previous studies that have dealt with L2 learners' general reading (e.g., Horiba, 1996; Lau, 2006). However, by using published research papers as reading texts, our study revealed several important findings. For example, we found that the less proficient student read from the beginning to the end and did not change his reading speed or depth of processing, while the proficient reader checked the sections and organization of the paper first before reading it again in detail.

Second, regarding the relationship between reading strategies and moves, both the proficient reader and the less proficient reader made many comments relating to Move 1 (topic/review) and Move 3 (outline/major findings). Furthermore, the less proficient reader processed Moves 1 and 3 in a similar way (Local $>$ Global $>$ Metacognitive) as the proficient reader processed the two moves in a similar way (Global $>$ Local $>$ Metacognitive). In other words, we could not find a significant interaction between reading strategies and the reading of particular moves. The relationship between reading strategies and moves in research papers has therefore yet to be clarified.

We have to acknowledge that there are some limitations to this study. The first regards the participants. It is true that, in previous studies focusing on L2 learners' academic reading and writing processes, it is not rare for the verbal protocols only of one or two participants to have been precisely analyzed (e.g., Cheng, 2008; Li \& Munby, 1996). Nevertheless, the number of participants in the present study was small. In the future, we need to accumulate data on analyses of the interaction between Swales' $(1990,2004)$ rhetorical "moves" and the reading strategies of more L2 learners. Furthermore, it will be important to control factors other than the focal ones. For example, our participant differed not only in L2 proficiency but also in EAP training; it will be necessary to compare strategy use among learners who are (a) less proficient and do not have explicit EAP training, (b) proficient without explicit EAP training, and (c) proficient with explicit EAP training. In addition, making a comparison between learners with different majors will also be useful in comprehending EFL learners' academic reading strategies.

Another limitation is concerning materials. Since the materials used in this study did not include enough text exemplifying Move 2 (identifying gaps in previous work), it will be necessary to conduct a similar study using empirical papers with Introduction, Method, Results, and Discussion organization or materials exhibiting more Move 2. As another future direction, we could conduct a think-aloud protocol using one research paper as a 
material. As we discussed in the Method section above, like those in previous studies (e.g., Cheng, 2008; Wyatt et al., 1993), our participants chose their own materials. We believe that this choice was right for the current participants considering that their majors were statistics and economics; however, in a general sense, it would be more convincing if we were able to achieve similar results by having participants read the same paper.

The third limitation we wish to mention is that the data analyzed in this study was limited. Assessing strategy use in reading a whole paper or a longitudinal multicase study will also help us to better understand the academic reading process. We are planning to incorporate these factors in our future studies.

Despite the above-mentioned limitations, we have obtained several pedagogical implications that will be useful for EFL academic courses at the undergraduate and graduate levels. First, since less proficient readers have difficulties with word-level processing, the use of English for specific purposes (ESP) and EAP corpora will be useful in improving and assessing the progress in their ability. Especially for undergraduate students and master's students who do not have much background knowledge, not knowing technical terms makes their reading extremely inefficient. Therefore, an ESP corpus needs to be developed for each discipline. The EAP corpus should put more emphasis on phrases frequently used in academic papers, since once they are familiar they can decrease the cognitive burden and increase the processing. For example, a frequently used phrase such as the purpose of this study is should be learned as one chunk. Second, in order to distinguish more and less important parts of papers, attention to metadiscourse (e.g., Williams, 2007) would be helpful. Metadiscourse is used to catch the reader's attention, so learning the meanings of types of metadiscourse will make it easier to grasp the writer's intention. Likewise, it is desirable for instruction in paragraph composition to be combined with reading instruction. It was demonstrated by the proficient reader in our study that instructions on how to develop a paragraph structure in academic writing help learners read papers more efficiently, because they use text and paragraph structure to more effectively skim passages. Considering this point, training in genre analysis (e.g., Swales, 1990, 2004) should also be useful, not only for writers but also for readers.

In conclusion, we found that our proficient learner read some parts carefully and skimmed other parts, while the less proficient learner read through the paper in a uniform fashion. The proficient reader used Global Strategies more than Local Strategies, while the less proficient reader used Local Strategies more than Global Strategies. In order to help the less proficient reader use more Global Strategies when appropriate, explicit instruction in text structure will be essential.

\section{References}

Bernhardt, E. (2005). Progress and procrastination in second language reading. Annual Review of Applied Linguistics, 25, 133-150. http://dx.doi.org/10.1017/S0267190505000073

Block, E. (1986). The comprehension strategies of second language readers. TESOL Quarterly, 20(3), 463-494. http://dx.doi.org/10.2307/3586295

Block, E. (1992). See how they read: Comprehension monitoring of L1 and L2 readers. TESOL Quarterly, 26(2), 319-344. http://dx.doi.org/10.2307/3587008

Carrell, P. (1989). Metacognitive awareness and second language reading. Modern Language Journal, 73(2), 121-133. http://dx.doi.org/10.1111/j.1540-4781.1989.tb02534.x

Cheng, A. (2008). Analyzing genre exemplars in preparation for writing: The case of an L2 graduate student in the ESP genre-based instructional framework of academic literacy. Applied Linguistics, 29(1), 50-71. http://dx.doi.org/10.1093/applin/amm021

Cogmen, S., \& Saracaloglu, A. S. (2009). Students' usage of reading strategies in the faculty of education. Procedia Social and Behavioral Sciences, 1, 248-251. http://dx.doi.org/10.1016/j.sbspro.2009.01.045

Dewey, D. P. (2004). A comparison of reading development by learners of Japanese in intensive domestic immersion and study abroad contexts. Studies in Second Language Acquisition, 26(2), 303-327. http://dx.doi.org/10.1017/S0272263104262076

Ericsson, K. A., \& Simon, H. A. (1984). Protocol analysis: Verbal reports as data. MA: MIT Press.

Gilboa, I. (2010). Questions in decision theory. Annual Review of Economics, 2, 1-19. $\mathrm{http}: / / \mathrm{dx}$.doi.org/10.1146/annurev.economics.102308.124332

Grabe, W. (2009). Reading in a second language: Moving from theory to practice. Cambridge University Press.

Grabe, W., \& Stoller, F. L. (Eds.). (2002). Teaching and researching reading. Harlow, UK.: Longman.

Green, A. (1998). Verbal protocol analysis in language testing research: A handbook. Cambridge University 
Press.

Halliday, M. A. K. (1994). An Introduction to functional grammar. London: Arnord.

Halliday, M. A. K. (2004). The language of science. New York: Continuum.

Halliday, M., \& Hasan, R. (1976). Cohesion in English. London Longman.

Hijikata, Y., Nakatani, Y., \& Shimizu, M. (2011). How Japanese EFL Learners Read Academic Papers? Paper presented at the 2011 Hawaiian Conference on Education in Honolulu, Hawaii.

Hijikata, Y., \& Nakatani, Y. (2010). The process of reading academic papers by Japanese EFL Learners: A think-aloud protocol study. Studies in Liberal Arts and Sciences, Tokyo University of Science, 43, 293-310.

Horiba, Y. (1996). Comprehension processes in L2 reading. Studies in Second Language Acquisition, 18(4), 433-473. http://dx.doi.org/10.1017/S0272263100015370

Johnson, P. (1992). Cohesion and coherence in compositions in Malay and English. RELC Journal, 23(2), 1-17. http://dx.doi.org/10.1177/003368829202300201

Kamhi-Stein, L. D. (2003). Reading in two languages: How attitudes toward home language and beliefs about reading affect the behaviors of "underprepared" L2 college readers. TESOL Quarterly, 37(1), 35-71. http://dx.doi.org/10.2307/3588465

Koda, K. (2005). Insights into second language reading. Cambridge University Press.

Lau, K-L. (2006). Reading strategy use between Chinese good and poor readers: A think-aloud study. Journal of Research in Reading, 29(4), 383-399. http://dx.doi.org/ 10.1111/j.1467-9817.2006.00302.x

Li, S., \& Munby, H. (1996). Metacognitive strategies in second language academic reading: A qualitative investigation. English for Specific Purposes, 15(3), 199-216.

Moore, N. A. J. (2006). Aligning theme and information structure to improve the readability of technical writing.Technical Writing and Communication, 36(1), 43-55.

Nassaji, H., \& Geva, E. (1999). The contribution of phonological and orthographic processing skills to adult ESL reading: Evidence from native speakers of Farsi. Applied Psycholinguistics, 20(2), 241-267.

Nassaji, H. (2003). Higher-level and lower-level text processing skills in advanced ESL reading comprehension. Modern Language Journal, 87(2), 261-276. http://dx.doi.org/10.1111/1540-4781.00189

Negretti, R., \& Kuteeva, M. (2011). Fostering metacognitive genre awareness in L2 academic reading and writing: A case study of pre-service English teachers. Journal of Second Language Writing, 20(2), 95-110. http://dx.doi.org/ 10.1016/j.jslw.2011.02.002

Paul, D., \& Charney, D. (1995). Introducing Chaos (Theory) into Science and Engineering: Effects of Rhetorical Strategies on Scientific Readers. Written Communication, 12(4), 396-438.

Phakiti, A. (2003). A closer look at the relationship of cognitive and metacognitive strategy use to EFL reading achievement test performance. Language Testing, 20(1), http://dx.doi.org/10.1191/02655322031t243oa

Phakiti, A. (2008). Construct validation of Bachman and Palmer's (1996) strategic competence model over time in EFL reading tests. Language Testing, 25(2), 237-272. http://dx.doi.org/10.1177/0265532207086783

Plakans, L. (2009). The role of reading strategies in integrated L2 writing task. Journal of English for Academic Purposes, 8(4), 252-266. http://dx.doi.org/10.1016/j.jeap.2009.05.001

Prentice, R. L., \& Pyke, R. (1979). Logistic disease incidence models and case-control studies. Biometrika, 66(3), 403-411.

Shiotsu, T., \& Weir, C. J. (2007). The relative significance of syntactic knowledge and vocabulary breadth in the prediction of reading comprehension test performance. Language Testing, 24(1), 99-128. http://dx.doi.org/10.1177/0265532207071513

Swales, J. M. (1990). Genre analysis. New York: Cambridge University Press.

Swales, J. M. (2004). Research genre. New York: Cambridge University Press.

Urquhart, S., \& Weir, C. (1998). Reading in a second language: Process, product and practice. Essex, U.K.: Addison Wesley Longman.

Williams, J. (2007). Style: Lessons in clarity and Grace (9th ed.). New York: Pearson Education, Inc. 
Wyatt, D., Pressley, M., El-Dinary, P. B., Stein, S., Evans, P., \& Brown, R. (1993). Comprehension strategies, worth and credibility monitoring, and evaluations: Cold and hot cognition when experts read professional articles that are important to them. Learning and Individual Differences, 5(1), 49-72. http://dx.doi.org/10.1016/1041-6080(93)90026-O 